\title{
Immune-Related Response Criteria Progressive Disease
}

National Cancer Institute

\section{Source}

National Cancer Institute. Immune-Related Response Criteria Progressive Disease. NCI

Thesaurus. Code C148196.

Increase in tumor burden greater than or equal to 25 percent relative to nadir (minimum recorded tumor burden); confirmation by a repeat, consecutive assessment no less than 4 weeks from the date first documented. 